\title{
Was ist das eigentlich: Projektunterricht? - Ein fiktives Gespräch über eine vage Idee
}

\section{Michael Schart}

\section{Einleitung}

Die Debatte um die Frage, die dem folgenden Beitrag seinen Titel gibt, ist so alt wie die Projektidee selbst. Schließlich kamen bereits Dewey und Kilpatrick, die gemeinhin als Väter des Gedankens in einem Atemzug genannt werden, keineswegs zu einer einheitlichen Definition der Sache (vgl. Magnor 1976). Mit ihrer Auseinandersetzung um das Rollenverständnis von Lehrenden und Lernenden im Projektunterricht haben sie eine Diskussion angestoßen, aus der beständig neue Interpretationsvarianten hervorgingen und die bis heute anhält. Und erst dieser Vagheit verdankt die Idee wohl ihre Langlebigkeit, trug doch die inhaltliche Unbestimmtheit entscheidend dazu bei, daß sie immer wieder als provozierend und zugleich auch inspirierend empfunden werden konnte (Oelkers 1997: 22). Tatsächlich überstanden Projekte alle didaktischen Strömungswechsel des vergangenen Jahrhunderts, bildeten den Kern vieler reformerischen Aktivitäten und haben sich bis heute ihren innovativen Charme bewahrt.

Daher erscheint es auch durchaus nicht nur beklagenswert, daß derart viele Definitionen kursieren. Gleichwohl möchte ich diesen mit dem folgenden Text keine eigene hinzufügen, denn das Thema hat meines Erachtens nach empirische Forschungen weitaus nötiger als weitere theoretische Erörterungen. Um auf die eingangs genannte Frage eine Antwort zu geben, werde ich deshalb keine theoretischen Argumente bemühen. Vielmehr habe ich eine Perspektive gewählt, die in den Diskussionen um den Projektunterricht bislang weitgehend vernachlässigt wurde: die der Lehrenden.

Im Zentrum des Beitrags steht ein Gespräch $^{1}$ zwischen zwei Lehrenden, die sich über ihre sehr unterschiedlichen Sichtweisen dieser Unterrichtsform austauschen. Obschon es sich bei ihnen um zwei fiktive Personen handelt, verstehe ich diesen Text aber nicht als eine Fiktion, sondern als einen von vielen sinnvollen Ansätzen, empirische Daten zu handhaben.

Die Grundlage dieses fiktiven Gespräches bilden Interviews mit dreizehn leibhaftigen Lehrenden für Deutsch als Fremdsprache, die ich im Rahmen einer qualitativen Studie zu ihren persönlichen Erfahrungen mit Projekten im DaF-Unterricht, insbesondere jenem im Rahmen der universitären Sommerkurse an deutschen Universitäten, befragte ${ }^{2}$. Der folgende Text ist daher Bestandteil einer

1 Für eine ausführlichere Version dieses Gespräches siehe Schart (2003).

2 Aus Platzgründen muß ich an dieser Stelle darauf verzichten, die Datenproduktion und -analyse in dieser Studie eingehender vorzustellen. Diese Informationen sowie sämtliche Forschungsinstrumente und alle Daten finden sich bei Schart (2003).

Info DaF 30, 6 (2003), 576-593 
weitaus umfassenderen Datenanalyse, besitzt jedoch - wie ich glaube - auch für sich selbst Erklärungswert und kann zu einem besseren Verständnis der Thematik beitragen.

Ein fiktiver Disput in der Tradition des »ethnographischen Dramas" (Richardson 2000: 934) erscheint mir vor allem deshalb zweckdienlich zu sein, weil sich mit ihm konträre Standpunkte besonders gut verdeutlichen lassen. Diese Darstellungsweise erleichtert es, die Divergenzen und Konflikte aufzuzeigen, die sich aus der Analyse der Daten ergeben. Freilich ist diese Form der Datenaufbereitung in der Fremdsprachenforschung (noch) nicht alltäglich, weshalb ich ihr einige Überlegungen zur Methodologie voranstellen möchte.

\section{Einige Bemerkungen methodologi- scher Art}

\subsection{Die Krise der Legitimation}

Die Einsicht, daß menschliches Wahrnehmen, Erkennen, Verstehen und Erklären nicht die Wirklichkeit selbst erfaßt, sondern »Bilder und Konstruktionen von ihr entwirft, diese Konstruktionen für wirklich hält, als Wirklichkeit definiert und sich an ihnen orientiert « (Soeffner 1999: 39), war für die Geistes- und die Sozialwissenschaften des 20. Jahrhunderts zweifellos eine äußerst folgenschwere. Früher oder später kamen Wissenschaftlerinnen und Wissenschaftler nämlich nicht umhin, diesen Gedanken auch auf ihre eigene Tätigkeit anzuwenden. Und das wiederum stürzte Disziplinen wie die Ethnographie oder die Soziologie geradewegs in eine $\gg$ Krise der Legitimation " (Gergen/Gergen 2000: $1020 \mathrm{f}$.). Denn wenn Forschende letztlich selbst nur Konstruktionen von Wirklichkeit liefern, können sie keine privilegierte Position beim Deuten sozialen Geschehens mehr beanspruchen und müssen andere Erkenntnisstile zumindest anerkennen. Es erscheint fast unumgänglich, daß bei einer solchen Sicht des empirischen Forschungsprozesses die Grenzen zwischen Wissenschaft und Fiktion verschwimmen, denn der Punkt, an dem eine beschreibende Abbildung in eine Einbildung des Autors umschlägt, kann nicht eindeutig bestimmt werden. Wird aber erst einmal bezweifelt, daß Forschungsmethoden und -techniken einen sicheren Pfad zur Wahrheit ebnen, liegt es eigentlich nahe, ins andere Extrem zu verfallen und anzunehmen, daß sich die Sozialwissenschaften eigentlich nur aus Wörtern und Symbolen zusammensetzen. Doch Forschende können einen Weg zwischen Szylla und Charybdis finden, wie Kemmis/McTaggart (2000: 580) es beschreiben, wenn sie es lernen, mit der Unsicherheit ihrer Erkenntnis zu leben, wenn sie akzeptieren, daß jede Wahrheit von bestimmten Interessen und historischen Bedingungen begrenzt wird, wenn sie diese Begrenzungen der eigenen Arbeit offenlegen, wenn sie ihre Forschungspraktiken und Urteile systematischem Zweifel unterziehen und sie sich der Intersubjektivität ihrer Deutungen versichern. Es gibt demnach vielfältige Möglichkeiten, um trotz des "Gewißheitszerfalls « (Reichertz 1999: 321) nicht der Beliebigkeit das Wort zu reden oder sogar in Lethargie $\mathrm{zu}$ verfallen. Und ein verändertes Verhältnis zur wissenschaftlichen Textproduktion gehört meines Erachtens nach unbedingt hinzu.

\subsection{Schreiben als Forschungsmethode}

Der wissenschaftliche Schreibprozeß erscheint in einem neuen Licht, sobald man ihn nicht mehr als bloße Reproduktion von Vorgefaßtem versteht, sondern vielmehr selbst als eine Methode betrachtet, um Wissen zu schaffen, Neues zu erkennen und zu entdecken: »By writing diffe- 
rent ways, we discover new aspects of our topic and our relationship to it. Form and content are inseparable«. (Richardson 2000: 923) Eine solche Sichtweise stellt die Zerrissenheit in Frage, die seit der Geburt der modernen Wissenschaften die Welt des Schreibens prägt. "Seit Kopernikus in den Himmel geschaut hat, leben wir in zwei Sphären. Da ist die Sphäre, in der man messen und rechnen kann, und da ist die Sphäre, in der man erleben und werten kann.« (Fischer 2001: 62)

»Fakt« und »Fiktion « müssen sich aber nicht notwendigerweise als derart unüberbrückbare Widersprüche gegenüberstehen, vor allem dann nicht, wenn die ausschlaggebende Differenz zwischen wissenschaftlichen und fiktiven Texten nicht mehr an der Frage entstehen kann, ob sie die Realität widerspiegeln oder nicht. So wie Schriftstellerinnen und Schriftsteller können jedenfalls auch Forschende bestrebt sein, mit ihren Texten menschliche Erfahrungen in eine Form $\mathrm{zu}$ übertragen, die es anderen erlaubt zu partizipieren (Bochner/Ellis 1996: 22).

Nach einer Phase der Verunsicherung hat sich deshalb in der qualitativen Forschung ein neues Bewußtsein für die enge Verwandtschaft von wissenschaftlichen und fiktiven Texten entwickelt und es entstanden vielfältige kreative Praktiken bei der Analyse von Daten - poetische und dialogische Darstellungsformen, Performance-Texte, fiktive Erzählungen u.a.m. (z.B. Blades 1997; Ellis/ Bochner 2000; Haggerson 2000; Huber 2000; siehe auch die Sammelbände von Denzin/Lincoln 2002 und Tierney/Lincoln 1997).

Allerdings - und das macht einen ihrer Unterschiede zum literarischen Schaffen aus - trifft die Wissenschaft bei diesem Unternehmen an Grenzen, die ihr von empirischen Daten gesetzt werden (Kellner/Heuberger 1999: 94). Um diese Ein- schränkung zu beschreiben, unterscheidet Reichertz (1999: 332) zwischen fiktiven und fiktionalen Texten. Erstere sind für ihn »Entwürfe mit besseren Gründen« bzw. »begründbare Als-ob-Unterstellungen, daß sich etwas so verhält, wie ich es unterstelle«, letztere dagegen solche mit "guten ästhetischen Gründen" bzw. »das mit einer freischwebenden Willkür Erfundene«.

Die Figuren unter ästhetischen Gesichtspunkten in einen Erzähltext einzubinden, stellt aus der wissenschaftlichen Perspektive kein maßgebliches Kriterium dar wenn es auch mit Blick auf die Lesbarkeit der Studie nicht vernachlässigt werden sollte. Entscheidender ist es, überzeugend und für Außenstehende nachvollziehbar zu erklären, wie diese Figuren konstruiert wurden, welchen Begegnungen und Beobachtungen sie entstammen. Insofern sind Forschende an empirische Daten gebunden, deren Entstehen sie reflektieren und dokumentieren müssen. Auch wenn sie dabei zu der Einsicht gelangen, daß diese Daten hoch selektiv und darüber hinaus unstabil sind, bedeutet das nicht, mit ihnen willkürlich verfahren zu können. »To make science more artful doesn't mean art and science become one and the same activity«. (Bochner/Ellis 1996: 22)

Es liegt also letztlich in den Händen der Autorinnen und Autoren selbst, ihre Werke in einem bestimmten Kontext zu verorten. Wenden sie sich als Forschende an die Öffentlichkeit, müssen sie anderen Bewertungskriterien gerecht werden als ihre literarisch ambitionierten Kolleginnen und Kollegen. Sie kommen beispielsweise nicht umhin, auch die Geschichten hinter der Geschichte zu erzählen, sich selbst erkennbar darzustellen, ihre eigenen Urteile in Zweifel zu ziehen und alle Daten der Öffentlichkeit zugänglich zu machen. 
Dennoch dürfen die »Figuren « in dem folgenden Gespräch nicht mit den Individuen selbst verwechselt werden. Als Konstrukte eines Forschungsprozesses ähneln sie tatsächlich Romanhelden. Es macht also schon angesichts der methodologischen Grundannahmen wenig Sinn, die Verwandtschaft zur Literatur abzustreiten. Die positiven Effekte dieser Beziehung herauszustellen, erscheint mir deshalb als die geeignetste Reaktion auf die Krise der Legitimation. Ich möchte einem Bild Benjamins (1980: 436) folgend - ein »nahrhaftes Gericht « zubereiten und es den Leserinnen und Lesern ermöglichen, sich die Erfahrungen anderer »einzuverleiben ", auch wenn die von mir arrangierte »appetitliche Ausstaffierung " nicht an jene heranreicht, wie sie literarische Texte darzubieten pflegen.

\section{Das Gespräch}

\subsection{Vorbemerkung}

Meine Interviewpartnerinnen und -partner gehen von einer vergleichbaren, wenn auch vagen Projektidee aus. Da sie diese aber auf sehr unterschiedliche Art und Weise in ihr berufliches Selbstverständnis integrieren, kommen sie zu einer jeweils besonderen Problemwahrnehmung und zu anderen Lösungsansätzen für die auftretenden Schwierigkeiten. Das folgende Gespräch soll diesen Prozeß an zwei Argumentationslinien verdeutlichen. Diese repräsentieren idealtypische und zugleich sehr gegensätzliche Positionen und stecken damit die Spielräume $a b$, innerhalb derer die konkreten individuellen Entwürfe meiner Interviewpartnerinnen und -partner ihre Gestalt annehmen.

Die Grundlage für die Konstruktion dieser beiden Argumentationslinien bilden die kodierten Segmente aus allen Interviewtexten (vgl. Schart 2003, CD-ROM). Indem ich diese Kodierungen zunächst nach Themenbereichen ordnete und dann auf Differenzen und Widersprüche befragte, ergab sich gleichsam das Drehbuch für eine fiktive Diskussion über die Möglichkeiten und Grenzen der Projektarbeit in den Sommerkursen.

Obschon sich dieser Meinungsaustausch zum größten Teil aus den Formulierungen meiner Interviewpartnerinnen und -partner zusammensetzt, hat dieser Text nicht zum Ziel, diese in dichotome Gruppen aufzuteilen. Es sind zuweilen auch die Zwiespalte innerhalb einzelner subjektiver Theorien selbst, die ich im Dialog aufgreife. Die Trennlinie verläuft in solchen Passagen also durch die betreffenden Lehrenden hindurch und die Darstellung nimmt eher den Charakter eines inneren Monologs an.

Lehrer X. und Lehrer Y., wie ich die beiden Gesprächspartner nennen möchte, widmen sich im ersten Dialogteil den erstrebenswerten Zielen der Projektarbeit. Der zweite Teil der Unterhaltung dreht sich um die Schwierigkeit, ein Projektthema effektiv zu bearbeiten und dabei als Lehrender eine angemessene Rolle $\mathrm{zu}$ finden. Im Mittelpunkt des dritten Teils schließlich steht die Frage, wie einzelne Projekte innerhalb einer Klasse verknüpft werden können und welche Verbindungen es darüber hinaus zum übrigen Geschehen des Sommerkurses geben sollte.

\subsection{Ziele}

X.: Unser Thema, der Projektunterricht, ist zwar ein beliebter Gegenstand der Didaktikausbildung, aber meines Erachtens nach auch eine Unterrichtsform, die sich sehr schwierig verwirklichen läßt.

Y.: Ja, das sehe ich ähnlich. Allerdings weiß ich nicht, ob du mit Projekten das gleiche meinst wie ich. Es gibt ja dazu sehr unterschiedliche Auffassungen. Unsere erste Frage dürfte also sein: Was verstehen wir eigentlich unter Projekten? 
Ich bin der Meinung, im Fremdsprachenunterricht zeichnen sie sich dadurch aus, $\mathrm{da} ß$ in ihnen die stringente, grammatikorientierte Sprachvermittlung in den Hintergrund tritt und sich das Geschehen statt dessen um andere Inhalte zentriert. Die Lernenden beschäftigen sich während eines bestimmten Zeitraumes in kleinen Gruppen und relativ selbständig zum Beispiel mit einem landeskundlichen Thema, sammeln dazu Informationen aus verschiedenen Quellen, arbeiten diese für die Präsentation vor der Klasse auf und wenden dabei ihre bisher erworbenen Kenntnisse kreativ an.

$X$.: Deiner Definition stimme ich zwar grundsätzlich $\mathrm{zu}$, aber sie erscheint mir zugleich auch unvollständig. Sie erklärt nämlich nicht, weshalb es überhaupt Sinn macht, die grammatische Systematik zugunsten anderer Inhalte zurückzustellen und die Selbständigkeit zu fördern. Ich glaube, die Antwort auf diese beiden Fragen hat unmittelbare Auswirkungen auf alle weiteren Überlegungen, und wir sollten deshalb an diesem Punkt ansetzen.

Y.: Wenn du so herangehen willst, müssen wir uns vielleicht erst einmal darüber verständigen, was wir uns generell vom Fremdsprachenunterricht versprechen, was er leisten soll und kann. Ich selbst sehe seine Aufgabe vor allem darin, Lernende mit systematischem Wissen auszurüsten, um eine Grundlage für den eigenständigen Spracherwerb zu schaffen. Ich glaube also, es ist wichtig, daß der Unterricht ganz systematisch aufgebaut ist. Deshalb fühle ich mich auch oft wie ein Gärtner, der behutsam kleine Pflänzchen zieht. Man muß ihnen Halt geben und sie hegen und pflegen, was eine Menge Ruhe und Geduld erfordert. Gerade in dieser Hinsicht finde ich die Sommerkurse übrigens problematisch. Ich bin nämlich nur eine kurze Zeit mit den Lernenden zusammen und die Vielfalt der unterschied- lichen Erfahrungen in einer Gruppe erschwert dieses systematische Lernen. Ich kann nicht auf einem gemeinsamen $\mathrm{Ni}$ veau aufbauen und Schritt für Schritt vorwärts gehen.

$X .:$ Ich finde, das ist eine sehr dominante Lehrerrolle, die du da beschreibst.

Y.: Sicher bin ich dominant. Was ist daran schlecht? Ich bin nun einmal in der Rolle des Lehrers und davon rücke ich auch kein Jota ab. Das bedeutet doch nicht, daß es langweilig für die Studierenden ist. Ich versuche immer, den Unterricht abwechslungsreich zu gestalten, zum Beispiel verschiedene Sozialformen und Medien einzusetzen. Das ist mir sehr wichtig. Aber eine Sprache lernt man nun mal, indem man Grammatik lernt und Vokabeln lernt. Und unsere Aufgabe liegt darin, diesen Prozeß anzuleiten. Wie ein guter Regisseur habe ich eben die Sache in der Hand, unter Kontrolle letztendlich. Ja, ich inszeniere eigentlich die Sprache. Die Herausforderung an uns sehe ich deshalb darin, daß wir sehr flexibel mit unserem Wissen umgehen und eine Progression an Grammatik und Vokabular bereitstellen müssen, die den jeweiligen Lernenden tatsächlich angemessen ist.

$X .:$ Und was bedeutet das alles für dein Konzept von Projektunterricht?

Y.: Wenn das systematische Lernen im Vordergrund steht, kommt der Beschäftigung mit fachfremden Inhalten natürlich nur eine zweitrangige Bedeutung zu. Da trenne ich schon sehr genau. Ich frage mich deshalb eigentlich auch, ob wir bei der Projektarbeit überhaupt von Lernen sprechen sollten. So wie ich meine Aufgabe als Lehrer sehe, erreiche ich einen wirklichen Lerneffekt eher durch gut strukturierten Unterricht, zum Beispiel durch Arbeitsblätter zu einem grammatischen Problem. Und ich denke, viele Kolleginnen und Kollegen verzichten gerade deshalb auf Projektunterricht, weil sie am Ertrag der Sache zweifeln. Soweit gehe 
ich nicht, denn ich sage ja nicht, Projektarbeit sei generell nutzlos. Ich bin jedoch der Meinung, daß es für einen effektiven Fremdsprachenunterricht bessere Methoden gibt.

$X .:$ Und weshalb setzt du dann überhaupt Projekte ein?

Y.: Zunächst aus einer rein praktischen Erwägung. Viele der Teilnehmenden an den Sommerkursen besuchen Deutschland zum ersten Mal. Es ist wichtig, daß sie sich an die neue Umgebung gewöhnen und auch etwas vom Alltag mitbekommen. Dafür müssen sie auch mal raus aus dem Unterricht. Zudem haben Projekte eine große motivierende Funktion. Die Teilnehmenden können ihre bisher erworbenen Sprachkenntnisse anwenden, alle Fertigkeiten zusammen üben und sich dabei selbst testen. Projekte locken also die Kreativität der Leute heraus und bringen Abwechslung in den Unterricht. Die Teilnehmenden arbeiten gemeinsam in einer entspannten Atmosphäre und legen dabei ihre Hemmungen vor dem Sprechen ab. Meine Erfahrung ist, daß das den Lernenden unheimlich Spaß macht.

$X .:$ Aber warum willst du dann nicht von einem Lerneffekt sprechen?

Y.: Weil ich sprachliches Lernen dabei allenfalls als einen Nebeneffekt sehe. Es beschränkt sich auf neuen Wortschatz, und auch das gilt meiner Ansicht nach nur für die Leute der Mittel- und vor allem der Oberstufe. Ich finde deshalb eigentlich auch die Bezeichnung "Projektunterricht « nicht ganz korrekt. Es ist kein Unterricht der deutschen Sprache im eigentlichen Sinne.

$X .:$ Auf das Thema kommt es dir also nicht an?

Y.: Natürlich ist es wichtig, daß die Studierenden Interesse am jeweiligen Gegenstand haben. Ohne das kann es nicht vernünftig klappen. Aber das Thema spielt für mich trotzdem keine vorder- gründige Rolle. Projekte definieren sich für mich über den selbständigen Umgang mit der Fremdsprache. Genau genommen fallen auch kreative Schreibaufgaben in diesen Bereich oder der Kochkurs, den wir jedes Jahr anbieten, und sogar die Sketche, die die Teilnehmenden immer am Abschlußabend aufführen. Vom Thema her ist das also sehr flexibel, auch wenn es nicht immer gleich Projekt heißt. $X .:$ In gewisser Weise gebe ich dir da recht. Selbst wenn die Teilnehmenden beim Bäcker ein Brötchen einkaufen gehen, ist das Projektarbeit. Auch in Sommerkursen, in denen offiziell keine Projekte angeboten werden, finden sie deshalb trotzdem statt, nur eben unbemerkt von den Lehrenden. Ich finde jedoch, daß diese Definition etwas zu kurz greift. Das ist sozusagen die elementare Ebene. Wenn wir über Unterricht sprechen, können wir nicht da stehen bleiben. Es kann dann nicht nur einfach darum gehen, daß die Teilnehmenden ins Sprechen kommen.

Y.: Aber es ist doch schon sehr viel erreicht, wenn es uns gelingt, daß sie ihre Hemmungen verlieren und frei sprechen, und wir sie damit vielleicht sogar motivieren, nach der Rückkehr in ihre Heimatländer weiter zu lernen. Und schließlich erhalten sie dabei ja auch noch viele verschiedene Informationen $\mathrm{zu}$ einem Thema. Das ist natürlich auch ein Lernprozeß, wenn auch einer, der nicht unmittelbar mit dem Deutschunterricht zu tun hat.

X.: Für mich steht an erster Stelle, daß sie die Fremdsprache benutzen wie jeder andere Mensch auch: nämlich mit einem bestimmten Ziel. Das Sprechen funktioniert nicht als Selbstzweck. Das Besondere an Projekten ist für mich, daß wir diesen freien Gebrauch der Fremdsprache nicht nur ermöglichen, sondern ihn auch reflektieren. Wir regen also dazu an, über das Erlebte bewußt nachzudenken. 
Wir wollen zum Beispiel wissen, was ihnen da draußen passiert ist, und dann versuchen, das gemeinsam zu vertiefen und einzuordnen. In Projekten steckt meines Erachtens nach weitaus mehr Potential als das von dir genannte. Aber um das nutzen zu können, müssen wir die thematischen Inhalte ernst nehmen. Das Einholen von Informationen zum Beispiel kann zu einer unendlichen Beschäftigung werden, wenn es keinen Zweck und kein Ziel hat. Ginge es nur darum, daß Lernende sich Informationen $\mathrm{zu}$ einem Thema heraussuchen, dann bräuchten wir dazu keinen Unterricht. Das machen sie auch von sich aus. Ich finde es weitaus wichtiger, daß sie ein Thema als eine intellektuelle Herausforderung begreifen, der sie sich alleine oder in Gruppen stellen und deren Bewältigung dann reflektiert wird. Projektarbeit bedeutet in diesem Sinne für mich, Lernende zum Denken anzuregen. Wo, wenn nicht an der Universität, soll das denn sonst geschehen?

Y.: Geht das nicht über unsere Aufgabe und auch unsere Möglichkeiten hinaus? Wir sind doch in erster Linie dazu da, um die Fremdsprache $\mathrm{zu}$ unterrichten. Du weißt selbst, wie schwierig es ist, mit Lernenden auf unteren Niveaustufen ins Gespräch zu kommen. Die können kaum etwas über sich selbst sagen und brauchen erst mal elementare Spracharbeit. Dein Konzept von Projekten wäre dann vor allem etwas für die Oberstufe, oder? Dann sind wirklich solche Projekte möglich, die ich eher als Untersuchungen bezeichnen würde. Insofern stimme ich dir zu. Aber das ist dann auch eine völlig andere Form von Zusammenarbeit. Dann bin ich nämlich aus der Verantwortung entlassen, die natürlich unnatürliche Situation des Fremdsprachenunterrichts ständig antizipieren und gestalten $\mathrm{zu}$ müssen. Bis die Lernenden die Oberstufe erreicht haben, trage ich eine bestimmte
Verantwortung für ihren Lernprozeß, die aus meinem Wissensvorsprung erwächst. Die läßt sich nicht so einfach hintergehen. Ich kann mich höchstens dumm stellen.

X.: Vielleicht sollte ich meinen Ausgangspunkt noch deutlicher erklären. Ich habe da vielleicht einen anderen Blick als du, denn ich komme eigentlich aus dem Bereich der Geschichte. Für mich spielt zum Beispiel die Landeskunde von vornherein eine viel größere Rolle als für viele meiner Kolleginnen und Kollegen, die mit den Methoden der Germanistik an den DaF-Unterricht herangehen und alle anderen Inhalte eher als etwas Zusätzliches begreifen. Die Blickrichtung ist also verschieden und deshalb gehen meine Überlegungen auch eher in Richtung projektorientiertes Arbeiten als in eine methodisch strukturierte Sprachvermittlung. Ich verstehe auch die von dir benutzte Metapher des Gärtners völlig anders. Einerseits leuchtet mir deine Interpretation natürlich ein: Das Sprachvermögen der Lernenden wächst allmählich wie ein Baum und wir helfen ihm dabei, indem wir ihn mit wohl dosierten Progressionen düngen und im Namen einer Methode oder eines Programms das Unkraut vernichten. Andererseits sehe ich mich aber immer wieder vor dem Problem, daß wir als Lehrende damit die Auswüchse nicht wirklich verhindern können. Es gelingt uns einfach nicht, all das Wuchern zu beschneiden. Das Lernpotential der Teilnehmenden ist heterogen und läßt sich nicht vereinheitlichen. Wir können höchstens versuchen, dem Wildwuchs gute Bedingungen zu schaffen. Nur in diesem Sinne fühle auch ich mich als Gärtner. Und den Projektunterricht empfinde ich dabei als besonders hilfreich, weil er für die Widerhaken sorgt, mit denen die Pflanzen Halt finden. Die Lernenden sehen sich anspruchsvollen Aufgaben gegenüber, de- 
ren Bewältigung sie in ihrem persönlichen Wachstum voranbringt. Und aus dieser Perspektive ist der Projektunterricht die absolut sinnvollste Methode.

Y.: Ich sehe noch nicht die Verbindung zum Fremdsprachunterricht. Unser Gegenstand ist die Fremdsprache. Das dürfen wir doch nicht aus den Augen verlieren.

$X .:$ Ich verstehe deine Bedenken. Zuweilen sind meine Projekte tatsächlich eher Geschichts-, Politik- oder sogar Biologieunterricht als Sprachunterricht. Das Erlernen der Fremdsprache erfolgt dabei, indem die Teilnehmenden sie gebrauchen, als ein Medium. Und das kann für die Einzelnen all die von dir genannten positiven Konsequenzen mit sich bringen: die Erfahrung, tatsächlich etwas mit der Fremdsprache zu bewirken, freier zu werden im Sprechen, Kontakte mit Deutschen zu knüpfen, Informationen sammeln. Und trotzdem denke ich, es muß doch etwas mehr dabei herauskommen. Das Sammeln von Informationen könnte man, wie gesagt, endlos betreiben. Auch Meinungsaustausch langweilt, wenn er nur zuliebe des Lehrers erfolgt. Das macht keinen Punkt. Es muß einen Stachel geben, der das Handeln in Gang setzt: Die Lernenden dringen experimentell in die Wirklichkeit ein, begreifen an einem kleinen Ausschnitt sozialer Realität größere Zusammenhänge, machen in der fremden Kultur eigene Entdeckungen und setzen dort vielleicht selbst etwas in Bewegung. Es geht mir also ausdrücklich nicht nur um den Spaß, auch wenn mir diese Form von Unterricht selbst sehr viel Spaß bereitet, ich gestehe es. Erst recht geht es mir nicht um neue Motivation für die nächste Übung zum Akkusativ oder so. Projektunterricht soll nicht in erster Linie Freude erzeugen, sondern etwas bringen: eine Erkenntnis, eine Erkundung, eine Reflektion des Erlebten. Er ist eine Herausforderung an die gesamte Persönlichkeit und hier liegt vielleicht der große Unterschied zu anderen Formen des Fremdsprachenunterrichts. Dadurch werden nämlich Kompetenzen gefördert, die normalerweise brachliegen. Lernende können sich mit ihren eigenen Stärken einbringen: der eine mit seiner sprachlichen Kompetenz, der andere mit seiner grammatischen Kompetenz und der dritte vielleicht mit seiner sozialen Kompetenz. Damit wächst die Chance, daß am Ende jeder das Gefühl hat, etwas Sinnvolles beigetragen zu haben. Auch das kann eine starke Motivation für das Weiterlernen sein.

Y.: In deiner Konzeption tritt also die Bedeutung der Fremdsprache weit hinter die anderer Lernziele zurück?

$X .:$ Projekte sind meines Erachtens nach auch dann sinnvoll, wenn die Lernenden, vor allem als Anfänger, auf ihre Muttersprache zurückgreifen, um eine Erfahrung festzuhalten oder kritisch aufzuarbeiten. Der Schritt in die Fremdsprache kann dann später immer noch erfolgen. Andererseits kann auch die Fremdsprache selbst zu einem Thema werden. Man kann zum Beispiel Projekte initiieren, bei denen die Leute den Gebrauch des Konjunktivs eigenständig recherchieren. Warum soll ich grammatische Themen immer als Fertiggericht vorsetzen? Wenn die Lernenden Grammatik wollen, kann man auch auf diese Weise ihren Wünschen nachkommen. Ich hoffe, du verstehst mich nicht falsch: Ich sage ja nicht, daß Projektunterricht ein Allheilmittel ist. Keine Lehr- und Lernform ist per se gut oder schlecht. Ich kann natürlich auch nach einem Buch vorgehen und gute Erfolge erzielen. Es gibt ja immer wieder Lernende, die mit sehr traditionellen Methoden tolle Ergebnisse erzielen. Aber vielleicht ist der Sommerkurs gerade dazu da, etwas anderes zu machen, die Defizite anzugehen, die auch 
diese Leute haben. Warum soll ich sie zum Beispiel mit der Grammatik und der oft schon veralteten Landeskunde von Lehrbüchern konfrontieren, wenn das natürliche Sprachumfeld rundherum ist? Wir sollten uns also zunächst fragen: Was kann man in vier Wochen Sommerkurs wirklich lehren? Und dann sehe ich eigentlich nicht, weshalb man übermäßig viel Grammatik anbieten sollte. Dazu brauchen die Leute doch nicht bis nach Deutschland zu kommen. Von daher kann ich auch gar nicht verstehen, daß man Projektunterricht nicht macht.

Y.: Ich habe da wirklich eine andere Perspektive. Ausgangspunkt ist für mich in jedem Fall die Frage, was mit einer bestimmten Unterrichtsphase erreicht werden soll. Und bei Projekten geht es mir nicht um strukturierte Sprachvermittlung, sondern um die kommunikative Erfahrung der Lernenden. Es ist für mich der ganze Sinn der Sache, daß die Teilnehmenden selbständig und kreativ in kleinen Gruppen agieren. Das macht den Hauptunterschied zum normalen Sprachunterricht aus. Aber man sollte das dosieren, denn die Beschäftigung mit anderen Themen ist eines von mehreren zusätzlichen Angeboten im Sommerkurs. Im Zentrum bleibt für mich eine gut strukturierte Sprachvermittlung. Sie ist für die natürlich unnatürliche Situation des Klassenzimmers einfach die effektivere Form des Lehrens.

$X .:$ Du siehst also eine Kausalität zwischen deinen Aktivitäten im Unterricht und dem Erfolg der Lernenden?

Y.: Natürlich glaube ich nicht, daß sie etwas beherrschen, nur weil sie es kennen. Aber wir müssen die besondere Situation akzeptieren, in der wir uns befinden. Der Sprachunterricht in einer Institution kann das Leben draußen nicht imitieren. Wir müssen unsere eigenen Möglichkeiten finden, die Fremdsprache effektiv zu vermitteln. Und unter diesem
Gesichtspunkt finde ich es unerläßlich, daß wir den Unterricht gut strukturieren und dann auch einzelne Lernziele überprüfen. Wir brauchen ja eine gewisse Rückmeldung darüber, wie viele von den Sachen angekommen sind, die wir vorbereitet haben.

$X .:$ Ich bin eher der Meinung, daß wir Lehrziele, wenn überhaupt, nur sehr langfristig überprüfen können. Das ist ja eigentlich das Verhängnisvolle an unserer Tätigkeit: wir können den Erfolg nicht richtig messen. Er manifestiert sich ja nicht in irgendwelchen Prüfungen. Manchmal ist es einfach nur die freundliche Resonanz, die mir Bestätigung und unheimlich viel neue Kraft gibt. Und manchmal glaube ich, man ist schon ein guter Lehrer, wenn man die Lernenden nicht behindert. Wir sind zwar häufig verpflichtet, einzelne Lehrziele zu überprüfen, aber wir sollten uns auch bewußt sein, daß wir mit dieser Art der Bewertung, unseren hochheiligen Prüfungen, den grassierenden Lehr-Lernaberglauben unterstützen. Aber das führt vielleicht zu weit weg von unserem Thema. $Y .:$ Ja, wir sollten uns genauer über die Probleme unterhalten, auf die wir bei der Projektarbeit stoßen. Angesichts unserer beiden unterschiedlichen Ansätze sind die sicher nicht gleich. Ich selbst finde es zum Beispiel immer wieder schwierig, geeignete Projektthemen $\mathrm{zu}$ finden und würde mir da schon ein paar mehr Anregungen erhoffen, zum Beispiel von Lehrwerken. Was da so vorgegeben wird, erscheint mir doch sehr simpel zu sein.

$X .:$ Die Schwierigkeit bei der Themenwahl besteht meines Erachtens nach darin, daß die Fragen, denen die Lernenden nachgehen sollen, nicht außerhalb ihrer Reichweite liegen dürfen. Das hemmt die Gestaltungsfreude von vornherein. Auch was wir Lehrende als spannende und politisch interessante Themen empfinden, muß bei den Lernenden überhaupt 
nicht so ankommen. Im Gegenteil, das kann den Unterricht ganz schön totschlagen. Entscheidend ist also die thematische Mit- und Selbstgestaltung durch die Lernenden.

Y.: Hast du nicht den Eindruck, daß die Mehrzahl der jungen Leute keine Lust auf anspruchsvolle Themen hat, und das selbst dann, wenn sie sprachlich schon sehr gut sind? In fließendem Deutsch können die zuweilen nichts sagen! Ich bin immer wieder überrascht, wie wenig Studierende dazu angeregt wurden, nachzudenken - und sei es über sich selbst.

$X .:$ Ich glaube auch, daß wir den kritischen Umgang mit Inhalten nicht voraussetzen können. Oft sind es sogar die vom Sprachniveau her schlechteren, die sich intellektuell beweglicher zeigen. Gerade deshalb ist es ja für mich eines der wichtigsten Ziele von Projekten, Denkmöglichkeiten zu schaffen.

$Y .:$ Dabei triffst du aber früher oder später auf das Problem, daß die Lernenden in Bereiche vordringen, in denen du dich selbst vielleicht nicht auskennst. Wir können schließlich nicht überall Experten sein. Fachmann bin ich zunächst einmal für mein eigenes Gebiet: die deutsche Sprache.

\subsection{Wege}

Y.: Neben dieser Unsicherheit in fachlichen Fragen stoße ich bei Projekten auch noch auf eine andere, eigentlich viel größere Schwierigkeit. Ich fühle nämlich immer eine Spannung zwischen meinem Anspruch, die Lernenden etwas selbständig tun zu lassen, und deren Fähigkeit, sich diese Freiheit auch wirklich zu nehmen. Bis die Mehrheit kapiert hat, worum es eigentlich bei der Projektarbeit geht, ist der halbe Kurs um. Also sage ich den Lernenden schon zu Beginn ganz klar, daß jetzt im Unterricht etwas völlig anderes passiert, als sie es vielleicht ge- wohnt sind. In gewisser Weise werfe ich sie damit natürlich ins kalte Wasser. Aber wenn die Sache erst mal läuft, das ist jedenfalls meine Erfahrung, dann dämmert ihnen meistens, worum es geht.

$X$.: Ich finde, es bringt kaum etwas, vorher viel theoretisch zu erklären oder sich vor die Klasse zu stellen und zu sagen: So wie ihr bisher gelernt habt, war völlig falsch. Jetzt zeige ich euch, wie es geht. Ich versuche statt dessen, das von einer inhaltlichen Idee ausgehend aufzubauen. Ich gebe zum Beispiel eine provozierende Frage in die Klasse und die Lernenden entwickeln sie weiter oder verändern sie, finden ihre eigenen Fragen. Dann kann man darüber sprechen, wo die Antwort vielleicht $\mathrm{zu}$ finden ist und wie man an sie herankommt. Und vor allem auch darüber, was in der gegebenen Zeit überhaupt möglich ist. Im Idealfall sollten Lernende die Möglichkeit bekommen, sich schrittweise an diese Form des Unterrichts $\mathrm{zu}$ gewöhnen. Man sollte ganz klein damit beginnen und erst mal danach fragen, welche Teile des Lehrbuchs sich durch eigenständiges Arbeiten oder durch Erkundungen außerhalb des Klassenraums ersetzen lassen. Das kann dann nach und nach ausgebaut werden. Ich glaube, sobald es die Lernenden gewohnt sind, mit verschiedenen Medien und in unterschiedlichen Sozialformen zu arbeiten, sind die Grundlagen schon geschaffen. Dann fehlt eigentlich nur noch eine Fragestellung als Motor, die das Projekt in Gang setzt.

Y.: Natürlich fände auch ich es viel besser, diese Arbeitsform ganz allmählich und implizit einfließen zu lassen, aber dann komme ich unweigerlich in Konflikt mit der sehr begrenzten Unterrichtszeit, die mir zur Verfügung steht. Mir fehlt genau dieser Spielraum und das erschwert die Projektarbeit gerade in den Sommerkursen ungemein. 
$X .:$ Ja, das empfinde auch ich als einen sehr problematischen Punkt an den Sommerkursen. Wenn Lernende keinerlei Erfahrung in selbständigen Lernformen mitbringen und anfangs noch dazu vielleicht sehr skeptisch sind, kann es schwierig werden. Ich kann mich für die Projektarbeit engagieren, ich kann versuchen, die Teilnehmenden davon zu überzeugen, daß auch dieses Lernen Erfolge bringt, aber ich kann niemanden zwingen. Und wenn jemand nicht will, dann sollten wir das auch akzeptieren.

Y.: Freiwilligkeit wäre also für dich eine wichtige Voraussetzung. Die Organisatoren in unserem Kurs sind dagegen der Meinung, man sollte den Teilnehmenden am Anfang des Kurses erklären, was Projektarbeit ist, dann die Themen kurz vorstellen und ihnen die Wahl lassen. Und sie müssen sich dann an einem Projekt beteiligen, sonst erhalten sie am Ende kein Zeugnis.

$X .:$ Ich finde es besser, Alternativen für diejenigen zu schaffen, die sich nicht zum Mitmachen bewegen lassen. Aber was mich an deiner Beschreibung noch mehr irritiert als der Zwang, ist der Zeitpunkt, zu dem bei euch Projekte eingeführt werden. Passiert das tatsächlich direkt zu Beginn des Kurses?

Y.: Ja, als ein Teil der Eröffnungsveranstaltung. Natürlich verstehen gerade die Lernenden auf den unteren Niveaustufen zunächst einmal überhaupt nicht, worum es geht. Die sitzen dann am ersten Projekttag zurückgelehnt im Klassenraum und denken, jetzt findet am Nachmittag eine Fortsetzung des Sprachunterrichts statt.

X.: Die Vorstellung, daß man versucht, Projektunterricht zu Kursbeginn in einem Hörsaal zu erklären, belustigt mich etwas. Das ist doch so ein sensibles Instrumentarium. Gerade wenn Lernende diese Form des Unterrichts nicht gewohnt sind, muß die psychologische
Ebene ernst genommen werden. Viele Lernenden sehen sich erst einmal vor dem Problem: Was will der jetzt eigentlich von mir? Und das kann Irritation, Verwirrung und Überlastung hervorrufen. Deshalb steht für mich am Beginn des Kurses ein Riesenberg an sozialer Arbeit. Sofort in ein Projekt einzusteigen, kommt meines Erachtens nach einem Blindflug sehr nahe. Zunächst müssen sich doch alle erst einmal zusammenfinden, so daß das nicht nur ein Haufen von Individuen ist, sondern wirklich eine Klasse. Das sind immer wieder relativ aufwendige Findungsprozesse. Sie müssen sich gegenseitig kennenlernen und beschnuppern. Sie müssen angstfrei miteinander umgehen - und auch mit mir. Meine Rolle ist zwar die des Lehrers, aber die sollen auch merken, da steckt ein Mensch dahinter. Dieses persönliche Verhältnis halte ich für besonders wichtig. Ich kann mir gar nicht vorstellen, wie man Projektunterricht beginnen kann, ohne daß es diese Beziehung zwischen allen Teilnehmenden gibt. Dann stehen auch die Chancen gleich viel besser, daß die Überzeugungsarbeit etwas bringt, denn Lust und Laune zu einem Projekt übertragen sich. Das schwappt zu ihnen über.

Y.: In unserem Kurs finden Projekte nur einmal pro Kurswoche statt. Da bleibt für diese Phase einfach keine Zeit. Die Teilnehmenden finden sich über ihr Interesse an einem Thema zusammen und arbeiten dann nur an den vier Nachmittagen gemeinsam. Und ich habe auch keine besonders negativen Erfahrungen mit dem persönlichen Verhältnis zwischen den Teilnehmenden gemacht. Mein Problem $\mathrm{zu}$ Beginn eines Projekts ist ein ganz anderes. Das Thema erscheint mir oft wie eine riesige Blase, in der die Lernenden ohne Orientierung umherschwimmen. Wie sollen sie auch überhaupt einen Weg finden, wenn sie das Ziel nicht kennen? 
Also muß ich mir vorweg Gedanken über die Ergebnisse machen. Deshalb bin ich es auch, der sich überlegt, wohin es überhaupt gehen soll. Ich muß also relativ klare Vorgaben entwickeln, ohne den Teilnehmenden die Möglichkeit zu nehmen, eigenständig zu sein. Das empfinde ich als eine besondere Herausforderung. Im normalen Unterricht kann ich auf Bekanntes zurückgreifen. Da passiert nichts umwerfend Neues. In Projekten besteht aber eine völlig andere Situation. $X .:$ Ja, unsere Rolle in den Projekten ist ambivalent. Auf der einen Seite wollen wir nicht, daß es in Beliebigkeit ausartet, auf der anderen Seite wollen wir aber auch nicht das Geschehen völlig dominieren.

Y.: Zumindest der Theorie nach sollen wir uns ja so wenig wie möglich in die Projektarbeit einmischen, aber das sehe ich wirklich differenzierter. Auch das ist eine pädagogische Situation. Ich bin kein primus inter pares. Ich bin der Lehrer. Und in dieser Funktion muß ich einen Vorlauf haben und das Projekt zumindest aus dem Hintergrund überblicken. In jedem Fall möchte ich verhindern, daß die Kursteilnehmer am Ende eines Kurses fragen: Was hatte das Projekt eigentlich für einen Sinn? Ich antizipiere also das Ergebnis und aus dieser Rolle komme ich auch nicht einfach raus. Wenn ich die Lernenden völlig alleine lasse, verlieren die sich vielleicht in dieser Blase und dann wird es schnell langweilig. Ich gebe ihnen also zunächst eine Orientierung und sage: etwa in diese Richtung sollte es gehen. Ich tue nicht etwa so, als hätte ich keine Ahnung davon, worauf es hinauslaufen soll, oder mache ihnen vor, daß alles ganz allein in ihrer Hand liegt. Das wäre doch ein Betrug an den Lernenden. $X .:$ Der entscheidende Unterschied zu anderen Unterrichtsformen liegt demnach für dich in der Art und Weise, wie
Lernende das von dir formulierte Ziel erreichen?

Y.: Wie ich bereits sagte: Für mich ist es der ganze Sinn der Sache, daß die Kursteilnehmer selbständig arbeiten und dabei wirklich einmal mit der Fremdsprache umgehen. Ich leiste die Vorarbeit, stelle die Weichen, halte mich dann aber aus der Gruppenarbeit weitestgehend raus. Das bedeutet ja nicht, daß ich schon vorher weiß, was zum Beispiel die Begegnungen mit Leuten auf der Straße bringen oder welche Informationen sie bei einer Recherche finden. Aber ich habe zumindest eine Vorstellung davon, wohin es geht. Das heißt auch nicht, daß ich den Teilnehmenden das Thema diktiere. Wir entwickeln das gemeinsam, aber dafür brauche ich erst mal selbst ein Konzept. Und gerade für den Sommerkurs mit seinem beschränkten Zeitvolumen fände ich deshalb sehr hilfreich, wenn es eine genauere Beschreibung der Projekte von seiten der Organisation gäbe. So etwas müßte eigentlich zur Vorbereitung des gesamten Kurses gehören, aber das fehlt bei uns leider völlig.

$X .:$ Ich folge dir nicht in allem, was du gesagt hast, stimme dir aber in einem Punkt völlig zu: Ein Thema auf eine Ebene herunter $\mathrm{zu}$ transformieren, auf der es mit den vorhandenen Mitteln bewältigt werden kann, das ist die entscheidende Aufgabe zu Beginn eines Projekts. Das ist eigentlich das Projekt! Jedes Thema bietet ja ganz viele Anknüpfungspunkte. Die Wirklichkeit ist konfus und der erste Schritt ist für mich deshalb, ein gemeinsames Verständnis der Fragestellung zu entwickeln. Wir müssen uns also in der Gruppe erst einmal darüber einig werden, was wir machen wollen und können. Ich bin dabei aber nicht derjenige, der ein festes Konzept präsentiert, sondern derjenige, der sich darum kümmern muß, daß sich die Vorschläge und Ideen der Studierenden realisieren las- 
sen. Ich versuche also, der Selbsttätigkeit die besten Bedingungen $\mathrm{zu}$ schaffen. Schließlich bin ich es, der die Gegebenheiten und die Mentalität der Leute hier kennt. Dann bei der praktischen Umsetzung der gemeinsamen Fragestellung, da habe ich oft das Gefühl, daß das auf einer gleichberechtigten Ebene geschieht, in einem für mich sehr angenehmen Klima. Von meiner Position aus sind aber die Ziele völlig offen. Natürlich gibt es auch Aufgabenstellungen, bei denen ich selbst mehr vorbereite, eine Stadterkundung zu Beginn des Kurses zum Beispiel. Doch bei größeren Projekten geht es oft in Richtungen, wo ich bekennen muß: Davon habe ich ja noch nie etwas gehört. Dann muß ich erst mal selbst nachforschen.

Y.: Du gehst also ohne festes Konzept in ein Projekt?

$X .:$ Natürlich mache ich mir auch meine Gedanken, aber ein wichtiger Effekt der Projektarbeit besteht doch gerade darin, daß die Kursteilnehmer es selbst lernen, ein Thema zu überblicken, in eine Struktur zu bringen und zu entscheiden, wie es sich bewältigen läßt. Ich kann ihnen dann Wege vorschlagen, aber diese lassen sich nicht vorplanen. Jedes Thema hat schließlich seine eigenen Lösungsschritte. Ich möchte sie auch nicht mit allen Mitteln vor der Erfahrung des Scheiterns bewahren. Auch das Ausweichen auf Alternativen führt nämlich $\mathrm{zu}$ wichtigen Lerneffekten. Und wenn es letztlich trotzdem keine Antwort gibt, ist das doch auch eine Entdeckung: Dann gibt es eben nichts.

Y.: Ich könnte es nicht verantworten, die Lernenden in Sackgassen laufen zu lassen. Du darfst nicht vergessen: Für unsere Projekte haben wir einen sehr begrenzten Zeitrahmen. Ich kann mir das vorstellen, wenn man über längere Zeit zusammen arbeitet. Dann kann Projektunterricht vielleicht auch einen anderen Stellenwert haben. Schon in unserer
Form war das ein Riesenaufwand für die Gruppen und mehr wäre mit Sicherheit zuviel geworden. Die kommen doch nicht nur nach Deutschland, um die ganze Zeit $\mathrm{zu}$ arbeiten. Also muß ich ihnen am Anfang zeigen, wie sie das Problem angehen können. Selbst wenn sie für das Thema Interesse haben, wissen sie häufig nicht, wie sie es bewältigen sollen. Dazu kommen die Probleme mit der Gruppenarbeit. Das Engagement ist sowieso unterschiedlich, aber wenn dann noch Unsicherheiten über das Vorgehen dazu kommen, kann das alles sprengen. Können wir es uns erlauben, Chaos zu erzeugen? Die Leute haben schließlich viel Geld für den Kurs bezahlt und wollen auch etwas dafür haben.

X.: Diese Unsicherheit, von der du sprichst, kann dann entstehen, wenn die Lernenden sich völlig selbst überlassen werden. Das ist natürlich nicht mein Ansatz. Es reicht eben nicht, ein Thema zu verabreden und dann $\mathrm{zu}$ sagen: nun macht mal! Projektarbeit bedeutet für mich auch, regelmäßig zusammenzukommen und zu fragen: Wo stehen wir? Was haben wir geschafft und was nicht? Warum hat etwas nicht geklappt? Wie könnte es anders gehen? Dann entsteht nicht das Gefühl, in ein Chaos zu geraten. Schwierigkeiten kommen freilich immer wieder vor, und trotz aller Sympathie für diese Unterrichtsform darf man diese Mißerfolge nicht verschweigen. Da verweigern sich zum Beispiel Gesprächspartner, da klappen Verabredungen nicht oder es klemmt bei der Gruppenarbeit. Projektunterricht bedeutet für mich deshalb, daß wir als Lehrende den Prozeß sehr sensibel begleiten und auf Stolpersteine achten. Wir müssen sie nicht in jedem Falle beiseite räumen, aber ihren Einfluß im Auge behalten. Ich sehe meine Aufgabe auch darin, die sozialen Prozesse in den Gruppen zu beobachten und $\mathrm{zu}$ thematisieren. Aber wieder sehr zu- 
rückhaltend. In jedem Menschen ist ja etwas, was man nicht erklären kann, und manche können eben nicht miteinander. Das muß man akzeptieren. Und darin steckt auch ein Lerneffekt, denn das auszuhalten gehört zum Leben. Das alles benötigt natürlich, da hast du recht, viel Zeit. Die muß die Kursgestaltung mir lassen. Das ist klar.

Y.: Und genau an diesem Punkt würde deine Konzeption in unserem Kurs schon scheitern. Wir bekommen diese Zeit nämlich nicht.

$X .:$ Wenn die Kursleitung unsere Arbeit nicht unterstützt, lassen sich Projekte, wie ich sie beschrieben habe, natürlich kaum verwirklichen. Die Offenheit von Projekten berührt ja letztlich auch die Grundstruktur des Kurses. Wenn Lernende eine kritische Kompetenz gegenüber ihrem Handeln aufbauen sollen, dann müssen wir sie auch als erwachsene Menschen anerkennen und ihnen Verantwortung übertragen. Und dann darf das Programm kein Hintergrundwissen von uns Lehrenden bleiben. Die Lernenden erfahren statt dessen, wie es ist, ein Programm zu gestalten. Sie machen mal das, was wir machen: Materialien suchen, Fragen aufwerfen und versuchen, ein Thema systematisch aufzuarbeiten. Projekte schaffen also Transparenz, und diese nicht zu gewähren, halte ich eigentlich für einen Betrug. Projekte sind in diesem Sinne eine Frage der Kurspolitik. Die Offenheit, die wir mit solchen Formen von Unterricht schaffen, kann sich über alle Elemente des Kurses ausdehnen, bis hin zur Leistungsbewertung am Ende. Wir sollten uns immer vor Augen halten: Die jungen Leute haben ihren eigenen Kopf und ihre eigenen Ideen und sie haben ein Recht darauf, als erwachsene Menschen behandelt zu werden.

Y.: Ich denke, in jeder Form von Unterricht gibt es eine Ungleichheit zwischen Lehrenden und Lernenden. Es gibt zum einen das Wissensgefälle und das läßt sich nicht wegreden. Und es gibt zum anderen die Verantwortung dafür, daß der Unterricht zu Ergebnissen führt. Die kannst du nicht einfach ablegen. Eine gewisse Führungsrolle wird von uns erwartet, und ich bin damit auch noch nicht auf Akzeptanzprobleme gestoßen. Die Selbständigkeit der Lernenden ist keine Sache, die unbedingt zu einem effektiven Unterricht gehören muß. Demgegenüber sehe ich aber sehr wohl die Gefahr, daß wir den Lernenden nur scheinbar Mitspracherecht einräumen, aber tatsächlich aus dem Hintergrund die Fäden in der Hand behalten. Dann finde ich es doch ehrlicher, die Ungleichheit zu akzeptieren. Und Transparenz schaffen wir ja zum Beispiel auch, indem wir den Lernenden das System des Deutschen offenlegen. Gerade für Erwachsene stellt das eine wichtige Abkürzung im Lernprozeß dar.

\subsection{Brücken}

$X .:$ Wir haben bisher über den Beginn und den Verlauf, aber noch nicht über das Ende eines Projekts gesprochen. Welche Rolle spielen für dich zum Beispiel Präsentationen?

Y.: Die Präsentationen dokumentieren die Ergebnisse der Gruppenarbeit und geben dem Projekt einen Abschluß. Jede Gruppe zeigt, was sie in den vier Wochen geleistet hat. Nicht zuletzt ergibt sich dadurch für alle eine schöne Erinnerung an den Kurs, denn jeder bekommt die gestalteten Materialien als Kopie mit nach Hause. Allerdings sehe ich gerade bei der Präsentation noch viel Veränderungsbedarf. In unserem Kurs passiert es sehr oft, daß Teilnehmende nach der Präsentation ihrer Ergebnisse enttäuscht sind. Sie haben sich sehr viel Mühe gegeben, aber die anderen Gruppen hören kaum zu, weil sie mit ihren eigenen Präsentationen beschäftig sind. Oft bleibt 
auch überhaupt keine Zeit mehr für Rückfragen, weil alles an einem Vormittag geschafft werden muß. Das frustriert natürlich.

X.: Diese Probleme kenne ich auch. Lernende erwarten Reaktionen auf ihre Präsentation. Sie brauchen dieses Erfolgserlebnis. Dafür muß aber die Präsentation selbst zu einer Lernsituation werden. Die Lernenden sollen formulieren, was ihnen gelang oder warum etwas nicht so wie erwartet lief. Das ist also die eine Seite: die Reflexion des eigenen Tuns. Die andere Seite ist die Wirkung der Präsentation auf das Publikum. Bei uns sollen die Präsentationen möglichst interaktiv gestaltet werden und alle Anwesenden einbeziehen. Allerdings muß ich gestehen, daß dieses Konzept bei uns noch nicht so gut klappt. Zunächst gibt es da häufig das Problem, daß nicht alle Erfahrung darin haben, Informationen interessant darzustellen. Dann erweist es sich immer wieder als Nachteil, wenn die Präsentationen an das Ende des Kurses gedrängt werden. Es wäre besser sie vorzuziehen und an verschiedenen Stellen einzubauen. Was wir aber nicht vergessen dürfen, wenn wir uns über die organisatorischen Einzelheiten unterhalten, ist eine grundlegende Frage: Warum sollen sich eigentlich die Kleingruppen für die Präsentationen der anderen interessieren? Eine Antwort wäre: weil diese Präsentation interessant gestaltet ist. Eine andere wäre jedoch: weil es ihre eigene Fragestellung unmittelbar betrifft.

Y.: Wie meinst du das?

$X .:$ Es müssen zunächst einmal gar nicht Gruppen sein, die in einer Präsentation ihre Ergebnisse austauschen. In der Arbeitsform bin ich sehr flexibel. Projektarbeit ist für mich kein Synonym zur Gruppenarbeit. Überhaupt nicht flexibel bin ich dagegen in der Frage des Themas. Wir machen nicht alle irgendwas, schon gar nicht das, was ich mir als Lehrer überlegt habe, sondern wir gehen gemeinsam dasselbe Thema an, wenn auch unter verschiedenen Gesichtspunkten. Jeder fügt dem Mosaik ein Steinchen hinzu, und damit verbessern sich natürlich automatisch die Chancen dafür, daß die Präsentationen nicht ins Leere laufen. Deshalb ist es auch so wichtig, zu Beginn eines Projekts ein gemeinsames Verständnis dafür zu entwickeln, worum es eigentlich gehen soll, welche Fragen wir angehen wollen und wie wir uns die Ergebnisse vorstellen.

Y.: Wenn man diese Überlegung weiterführt, könnten ja sogar alle Projekte in einem Sommerkurs irgendwie zusammenhängen. Oft gibt es für die Kurse ein bestimmtes Motto. Das wäre dann zum Beispiel ein Anknüpfungspunkt. Aber um diese Zusammenarbeit zu organisieren, bedarf es einer bestimmten Kursstruktur. Bei uns bieten alle Lehrenden ihr eigenes Projektthema an und deshalb fehlen diese Verbindungen zwischen den einzelnen Gruppen völlig. Die Kursleitung sagt uns: Ihr müßt ein Projekt organisieren und dann machen eben alle das, wofür sie sich selbst interessieren. Am Ende kommt der ganze Kurs wieder zusammen und jede Gruppe gibt eine kurze Vorstellung. Das ist zwar dann für uns Lehrende ganz interessant und lustig, aber die anderen Teilnehmenden im $\mathrm{Pu}$ blikum bekommen eigentlich wenig mit. Um das $z u$ ändern, müßten wir enger zusammenarbeiten und die Sache gründlicher vorbereiten. Aber die Chancen dafür stehen nicht sehr gut, denn bei uns gibt es einige Projektmuffel, die sagen, das sei sowieso alles neumodischer Käse und wenn sie es nicht müßten, würden sie gerne darauf verzichten. Bei manchen Kolleginnen und Kollegen wäre es sicher gut, wenn sie selbst noch mal in die Schule gingen, damit sie sich ein bißchen öffnen. Die haben ja selbst erst wenig Erfahrung darin, was Projektunterricht 
sein kann, und dann sind die Zweifel natürlich verständlich. Auch bei den Lernenden springt der Funke ja oft erst dann über, wenn sie sehen, wie Projektarbeit läuft.

$X .:$ Deshalb ist es in unserem Kurs auch so wichtig, daß wir einen festen Stamm von Lehrenden haben. Viele haben schon als studentische Tutoren angefangen und dort bei der Projektarbeit geholfen. Dann ist der Schritt natürlich nicht mehr so groß, wenn man selbst als Lehrer vor der Klasse steht. Wir merken das immer wieder bei Leuten, die von außen neu dazukommen. Denen fällt es viel schwerer, sich in diese Form des Unterrichtens einzufinden. Wie du schon sagtest: Wenn man das nicht mal erlebt hat, bleibt es sehr theoretisch.

Y.: Glaubst du eigentlich, daß es so etwas wie ein bestimmtes Persönlichkeitsprofil für den Projektunterricht gibt?

$X .:$ Engagement brauchen Lehrende nicht nur für den Projektunterricht, aber da wohl besonders. Es gehört sicher einiger Mut dazu, Kompetenzen abzugeben, auf die traditionellen Machtmittel des Unterrichtens wie Tafel, Methode oder die Unterteilung in hinten und vorne $\mathrm{zu}$ verzichten, ein gewisses Chaos auszuhalten und sich auch mal auf Gentechnik einzulassen. Prinzipiell kann das vielleicht jeder, aber man muß sich auch ganz bewußt dafür entscheiden. Vielleicht fällt mir das nicht so schwer, weil ich mich auch selbst noch als lernender Mensch begreife. Ich will ja auch etwas von den Lernenden, was mehr ist als eine richtige Antwort in einer Grammatikübung.

Y.: Ich denke auch, daß Lehrende in jeder Form von Unterricht mit dem Gedanken zurechtkommen müssen, jeden Augenblick in ein Loch fallen $\mathrm{zu}$ können. Um unter solchen Bedingungen $\mathrm{zu}$ arbeiten, bedarf es sicher eines bestimmten Persönlichkeitsprofils. Wenn man das hat, kann man meines Erachtens nach auch
Projektunterricht machen. Wenn nicht, ist man auch sonst kein guter Lehrer.

$X$. : Die institutionellen Rahmenbedingungen spielen natürlich auch eine wichtige Rolle. Die geistige Trägheit von Kursleitungen kann jede Experimentierfreude unglaublich einengen. Meines Erachtens nach bestimmt nicht zuletzt die organisatorische Struktur eines Kurses darüber, ob sich Räume für Neues öffnen. Viele der zweifelnden Kolleginnen und Kollegen sind ja bei solchen Veränderungen dabei, wenn sie sich auch wirklich eingebunden fühlen.

Y.: Aber wie macht ihr das? Wir kommen eigentlich nur für die Dauer des Kurses zusammen und wohnen sonst in anderen Städten und zum Teil sogar im Ausland. Das begrenzt die Möglichkeiten der Zusammenarbeit von vornherein und es befördert übrigens auch die Unsicherheit. Wer die Gegebenheiten vor Ort nicht genau kennt, kann den Lernenden in Projekten auch nicht in der Weise kompetent zur Seite stehen, wie du es beschreibst.

$X .:$ Das sind natürlich keine tollen Ausgangsbedingungen. Wie alles, was man gut machen will, braucht so ein Sommerkurs jede Menge Vorarbeit. Bei uns gibt es deshalb mehrere Vorbereitungstreffen, an denen alle Lehrende und auch die studentischen Hilfskräfte teilnehmen. Bei diesen Treffen entsteht eigentlich erst das Team und eine gute Atmosphäre ist uns ganz wichtig. Die wirkt nämlich auf die Lernenden ansteckend und macht vieles möglich. Der Kurs wird zu einem Gesamtkunstwerk, wie wir es gerne bezeichnen, in dem der Projektunterricht seinen festen Platz hat und mit den anderen Elementen eng verknüpft ist. Die genauen Absprachen vor Beginn des Kurses bringen den Vorteil mit sich, daß die Projekte als Teil des Curriculums von der Kursleitung akzeptiert werden. Ich muß also nicht schwitzend da sitzen und 
mich fragen, an welcher Stelle des Unterrichts ich das reinklemmen kann. Und die Studierenden bekommen dadurch das Gefühl, das gehört einfach dazu. Es steht im Kursprogramm und hat schon dadurch einen gewissen Autoritätscharakter. Wir bekommen also eine große Spielwiese, um solche Sachen in den Unterricht einzubauen.

Y.: Eine so intensive Vorbereitung in der Gruppe macht natürlich vieles einfacher. Aber was ist mit den Kursteilnehmern? Viele von ihnen wollen ja vor allem systematischen Unterricht. Ich habe jedenfalls die Erfahrung gemacht, daß die Lernenden zunächst einmal mit viel Skepsis auf Projekte reagieren. Sie akzeptieren sie im Hinblick darauf, daß sie neues Wissen zu einem bestimmten landeskundlichen Thema erwerben, und sehen sie als Möglichkeit, bisher Gelerntes anzuwenden. Aber sie erwarten weitaus mehr von unserem Unterricht. Sie wollen Grammatik üben, sie wollen eine genaue Kontrolle ihrer Einzelleistung und sie sind vor allem nicht nur hier, um zu lernen und ihre ganze Freizeit in Projekte zu investieren.

$X$. : Da gebe ich dir Recht. Viele der Teilnehmenden haben natürlich zunächst den Eindruck, bei der Projektarbeit weniger zu lernen. Das heißt, sie erkennen die Fähigkeit, ein Problem zu lösen, nicht als einen Lernerfolg an. Ich stelle natürlich diesen Lernaberglauben in Rechnung, versuche, ihren Anspruch zu befriedigen, und zeige, daß ich auch die deutsche Grammatik beherrsche. Aber ich versuche ihnen auch klarzumachen, daß es anders gehen kann. Jedenfalls akzeptiere ich die Lerntraditionen nicht als ein Argument, um vielleicht sogar ganz auf Projekte zu verzichten. Ich habe festgestellt, daß die, die einsehen, warum wir das machen und die Spaß an ihrem Thema haben, nach und nach auch mit der Arbeitsform zurechtkommen. Und diese Kompetenzen zu entwickeln, gehört für mich ja gerade zu einem wichtigen Ziel von Projekten. Insofern brauchen sie keine besonderen Voraussetzungen auf diesem Gebiet mitzubringen. Sie sind Lernende. Das reicht. Und sie kommen ja nicht nur mit ihren Lerngewohnheiten und ihrer Skepsis, die kommen doch auch mit der großen Erwartung, etwas Neues und Ungewohntes zu erleben. Dem müssen wir etwas entgegenhalten.

Y.: Grundsätzlich stimme ich dir natürlich $\mathrm{zu}$, aber meine Konsequenzen sind eben etwas anders. Wir kommen eben immer wieder auf unsere sehr verschiedenen Ausgangspositionen zurück.

$X .:$ Ja, wir haben in diesem Gespräch zu keinem gemeinsamen Konzept gefunden. Aber vielleicht hebt sich ja der Projektunterricht gerade durch seine NichtEinheitlichkeit von den Vereinheitlichungsbemühungen in der Didaktik ab. Alles, was keiner bestimmten Methodik folgt und sich auf das Geschehen einläßt, geht sicher schon in Richtung Projekt.

\section{Literatur}

Benjamin, Walter: »Denkbilder «. In: Walter Benjamin: Gesammelte Schriften. Band IV, 1. Frankfurt a. M.: Suhrkamp, 1980, 305438.

Blades, David W.: Procedures of power and curriculum change. New York: Lang, 1997.

Denzin, Norman K.; Lincoln, Yvonna S. (Hrsg.): Handbook of qualitative research. Second edition. Thousand Oaks: Sage, 2000.

Denzin, Norman K.; Lincoln, Yvonna S. (Hrsg.): The qualitative inquiry reader. Thousand Oaks: Sage, 2002.

Ellis, Carolyn; Bochner, Arthur P.: »Autoethnography, personal narrative, reflexivity: researcher as subject «. In: Denzin, Norman K.; Lincoln, Yvonna S. (Hrsg.): Handbook of qualitative research. Second edition. Thousand Oaks: Sage, 2000, 733768. 
Fischer, Ernst Peter: Die andere Bildung. Was man von den Naturwissenschaften wissen sollte. München: Ullstein, 2001.

Gergen, Mary M.; Gergen, Kenneth J.: "Qualitative inquiry: Tensions and transformations «. In: Denzin, Norman K.; Lincoln, Yvonna S. (Hrsg.): Handbook of qualitative research. Second edition. Thousand Oaks: Sage, 2000, 1025-1046.

Haggerson, Nelson L.: »A poetry of curriculum inquiry«. In: Haggerson, Nelson L.: Expanding curriculum research and understanding. A mytho-poetic perspective. New York: Lang, 2000, 219-224.

Hitzler, Ronald; Reichertz, Jo; Schröer, Norbert (Hrsg.): Hermeneutische Wissenssoziologie - Standpunkte zur Theorie der Interpretation. Konstanz: Universitätsverlag, 1999.

Huber, Andreas: Die Angst des Wissenschaftlers vor der Ästhetik. [34 Absätze]. Forum Qualitative Sozialforschung, 2001. [Online Journal] 2 (2). Verfügbar über: http/ / qualitative-research.net/fgs [Zugriff: 20.01.2003].

Kellner, Hansfried; Heuberger, Frank: »Die Einheit der Handlung als methodologisches Problem«. In: Hitzler, Ronald; Reichertz, Jo; Schröer, Norbert (Hrsg.): Hermeneutische Wissenssoziologie - Standpunkte zur Theorie der Interpretation. Konstanz: Universitätsverlag, 1999, 71-96.

Kemmis, Stephen; McTaggart, Robbin: »Participatory action research «. In: Denzin, Norman K.; Lincoln, Yvonna S. (Hrsg.): Handbook of qualitative research. Second edition. Thousand Oaks: Sage, 2000, 567-606.
Lehker, Marianne: »Projektarbeit im DaFUnterricht «, Info DaF 30,6 (2003), 562-575.

Magnor, Manfred: Die Projektmethode: Ein Ergebnis der philosophischen und erziehungstheoretischen Ansätze von John Dewey und William H. Kilpatrick. Universität Osnabrück, Dissertation, 1976.

Oelkers, Jürgen: »Geschichte und Nutzen der Projektmethode«. In: Hänsel, Dagmar (Hrsg.): Handbuch Projektunterricht. Weinheim: Beltz, 1997, 13-30.

Reichertz, Jo: »Über das Problem der Gültigkeit von Qualitativer Sozialforschung «. In: Hitzler, Ronald; Reichertz, Jo; Schröer, Norbert (Hrsg.): Hermeneutische Wissenssoziologie - Standpunkte zur Theorie der Interpretation. Konstanz: Universitätsverlag, 1999, 319-348.

Richardson, Laurel: »Writing: A method of inquiry «. In: Denzin, Norman K.; Lincoln, Yvonna S. (Hrsg.): Handbook of qualitative research. Second edition. Thousand Oaks: Sage, 2000, 923-948.

Schart, Michael: Projektunterricht - subjektiv betrachtet. Eine qualitative Studie mit Lehrenden für Deutsch als Fremdsprache. Hohengehren: Schneider, 2002.

Soeffner, Hans-Georg: »Verstehende Soziologie und sozialwissenschaftliche Hermeneutik «. In: Hitzler, Ronald; Reichertz, Jo; Schröer, Norbert (Hrsg.): Hermeneutische Wissenssoziologie - Standpunkte zur Theorie der Interpretation. Konstanz: Universitätsverlag, 1999, 39-50.

Tierney, William G.; Lincoln, Yvonna S. (Hrsg.): Representation and the text. Reframing the narrative voice. New York: State University of New York Press, 1997. 